\title{
Traveling wave resonant ring for electron cloud studies
}

\author{
U. Iriso, ${ }^{1}$ F. Caspers, ${ }^{1}$ J-M. Laurent,${ }^{1}$ and A. Mostacci $^{2}$ \\ ${ }^{1}$ CERN, CH-1211, Geneva 23, Switzerland \\ ${ }^{2}$ Università di Roma “La Sapienza,” Rome, Italy
}

(Received 31 July 2002; revised manuscript received 13 March 2004; published 26 July 2004)

\begin{abstract}
Within the framework of the CERN program on electron cloud effects in accelerators, a coaxial multipacting test stand was built. In order to simulate bunched beam, the test stand is subjected to short rf pulses. The field strength in a traveling wave mode is sufficient to trigger multipacting in "as received" surfaces, but not in chambers treated to reduce the secondary emission yield. Thus a number of upgrades in the bench setup have been pursued, mainly in two directions. The first one is a general reduction in mismatching (i.e., electrical losses) amongst the different parts of the setup. Secondly, instead of dumping the pulsed power into a load, it is recirculated by means of a broadband working regime resonant ring. This ring required the design of a directional coupler with up to $1 \mathrm{kV}$ dc isolation, very low transmission losses, and a four octave bandwidth. This paper reports on the steps required to build this traveling wave resonant ring (improvements on the chamber and implementation of the coupler) and includes an appendix on the main properties of the setup that relate to electron multipacting studies.
\end{abstract}

DOI: 10.1103/PhysRevSTAB.7.073501

PACS numbers: 29.27.Bd

\section{INTRODUCTION}

Multipacting is an electron multiplication resonance, which develops in $\mathrm{rf}$ devices when periodic field strength is maintained between two opposite surfaces and certain energy and resonant conditions for electron kinetics are met. According to Ref. [1], such conditions are expected to occur in the Large Hadron Collider (LHC) for the typical LHC parameters (Table I). The bunched proton beam will provide the periodic electric field. An electron cloud may develop that could cause vacuum breakdown, associated with a rapid pressure increase, that may lead to significant degradation in beam performance and/or excessive liquid helium consumption. In order to study those phenomena in a laboratory, a bench test setup [2] was built where six wires are inserted in a circular vacuum chamber and subjected to short $\mathrm{rf}$ pulses the transverse electromagnetic (TEM) field produced by a bunched beam (Fig. 1). As will be shown in Sec. II, six wires were used instead of one to improve the impedance matching. For a comparable single wire configuration the wire would need to be approximately $45 \mathrm{~mm}$ in diameter, thereby becoming an obstacle for the electrons. The six wire configuration is suitable for achieving the required impedance $(50 \Omega)$ through the chamber. On the other hand, the use of more than six wires would strongly perturb the electron path when multipacting occurs. See Ref. [2] for more details about the field topology and electron dynamics in this setup. The traveling wave (TW) coaxial structure is powered by a wide band power amplifier, ${ }^{1}$ which is driven by a pulse generator. Typically, the shape of the traveling pulse that produces

\footnotetext{
${ }^{1}$ Amplifier research, Model 100W1000, 1-1000 MHz, $100 \mathrm{~W}$.
}

the multipacting for this setup can, as a first approximation, be considered square. Its period ranges from 20 to $50 \mathrm{~ns}$, while its pulse length ranges from 10 to $20 \mathrm{~ns}$ (see Ref. [2] for more details). The output of the setup (upper part of the chamber; see Fig. 1) is connected to an rf load, which absorbs the transmitted power and prevents undesirable reflections. Two probes were installed to collect electrons, one placed on top of the chamber, the other in the middle of it, as shown in Fig. 1.

The achievable electric field strength is mainly limited by the output power of the wide band amplifier. The goal would be to reproduce exactly the pulse parameters shown in Table I. The available instruments let us reproduce the 25 ns pulse spacing $(\Delta T)$, but limit the minimum achievable pulse length $\left(t_{w}\right)$ to $3.1 \mathrm{~ns}$. On the $50 \Omega$ load, the initial (i.e., before improvements) output voltage circulating through the wires, $V_{W}$, is limited to $100 \mathrm{~V}$ (base line peak), for which electrons get an energy $E_{e}^{\text {in }}=75 \mathrm{eV}$, according to both simulations and measurements in Ref. [2]. The secondary emission yield (SEY) of a given material is defined as the number of secondary electrons emitted by its surface per impinging electron. The multipacting effect is possible if the ratio between the total number of impinging electrons and the generated electrons in the wall is greater than 1 . SEY is a function of the electron energy, and it is usually characterized by the maximum yield $\left(\mathrm{SEY}_{\max }\right)$ and the energy at which this maximum occurs $\left(E_{0}\right)$. Figure 2 shows two instances of SEY dependence on impinging electron energy for a copper surface before and after a process to reduce its SEY. As can be seen there, when a material is treated to reduce its $\mathrm{SEY}, \mathrm{SEY}_{\max }$ is decreased and $E_{0}$ is pushed higher. The material can be treated by baking it out, applying a wall surface coating to it, or exposing it to 
TABLE I. Nominal parameters for the LHC beam and chamber, and estimated proton intensity threshold to produce electron multipacting. Updates for these parameters can be checked at http://bruening.home.cern.ch/bruening/lcc/WWW-pages/nominal_parameter_ v3.html

\begin{tabular}{ccccc}
\hline \hline $\begin{array}{c}\text { Nominal } \\
\text { charge }\end{array}$ & $\begin{array}{c}\text { rms bunch length } \\
\text { (flattop) }\end{array}$ & $\begin{array}{c}\text { Bunch } \\
\text { spacing }\end{array}$ & $\begin{array}{c}\text { Average chamber } \\
\text { radius }\end{array}$ & $\begin{array}{c}\text { Multipacting } \\
\text { threshold }\end{array}$ \\
\hline $10^{11} \mathrm{p} /$ bunch & $0.25 \mathrm{~ns}$ & $25 \mathrm{~ns}$ & $20 \mathrm{~mm}$ & $2.3 \times 10^{10} \mathrm{p} / \mathrm{bunch}$ \\
\hline \hline
\end{tabular}

an electron dose [3]. Therefore, due to the interest in studying multipacting for these surfaces, it is desirable to reach higher electron multipacting energies using the new bench test stand. The dependence of the pulse amplitude required to trigger multipacting in the TW chamber as a function of electron dose is studied in Ref. [2], and the results are shown in Fig. 3. After the bakeout of a stainless steel surface and a small electron dose, the minimum pulse amplitude required to trigger multipact-

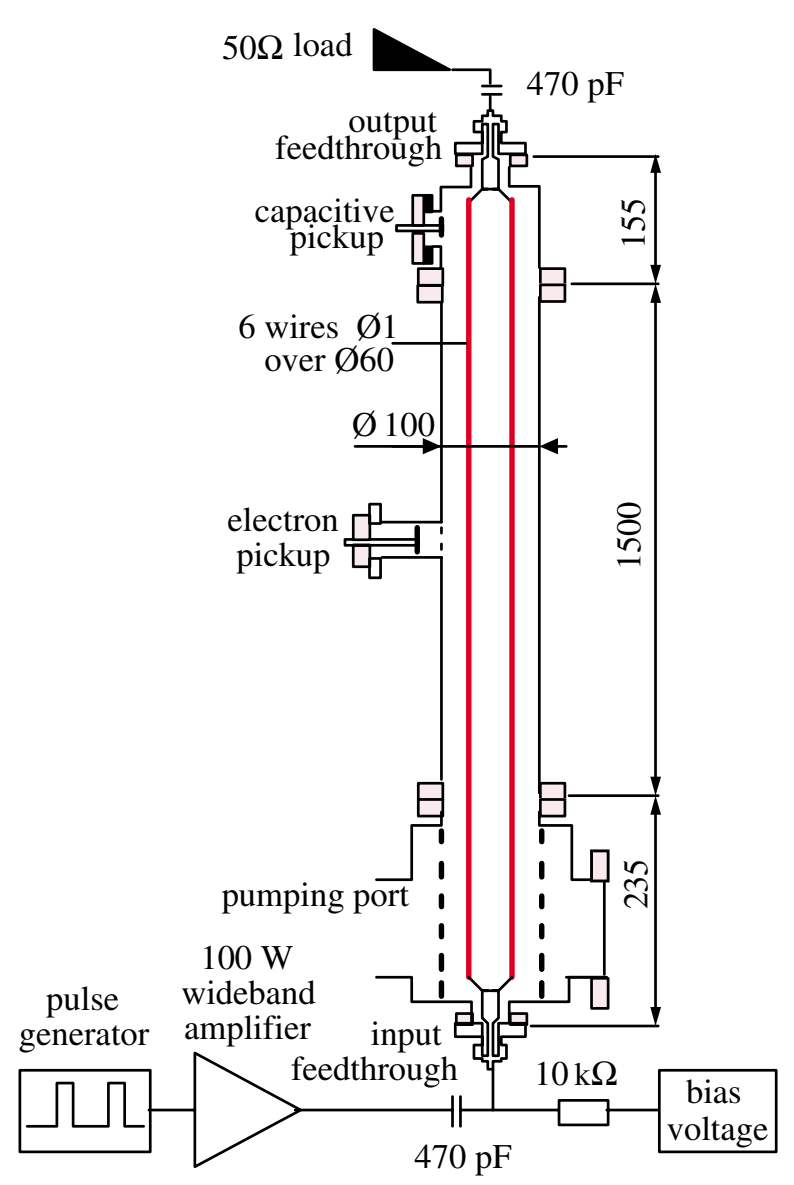

FIG. 1. (Color) TW multiwire chamber. The pulses coming from the signal generator are amplified by the wide band power amplifier. Each pulse, biased to ensure a positive voltage, travels along the six wires inside the chamber. The $50 \Omega \mathrm{rf}$ load on the top avoids undesirable reflections. Capacitors on the top and the bottom isolate the dc currents inside the chamber. Lengths are given in $\mathrm{mm}$. ing exceeds $200 \mathrm{~V}$. Therefore, higher traveling wave voltage $V_{W}$ (around $300 \mathrm{~V}$ peak-peak) is necessary.

One possible way to increase the voltage $V_{W}$ (without changing the amplifier) is to reinject a fraction of the output power into the system, which is similar to what was proposed in Ref. [4]. Such a recirculating scheme is called a resonant ring (RR) and allows a much more efficient use of the amplifier output power. Resonant rings (or ring resonators) at a single frequency have been

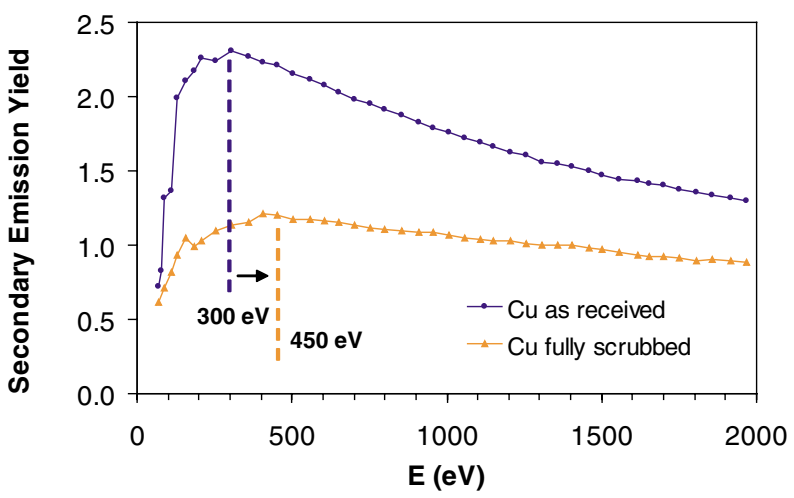

FIG. 2. (Color) SEY as a function of the electron energy for an "as received" copper surface (upper curve) and that surface after intense electron bombardment (lower curve). After electron bombardment, SEY is lower and SEY max $_{\text {occurs at higher }}$ electron energies. Courtesy of M. Pivi (Ref. [2]).

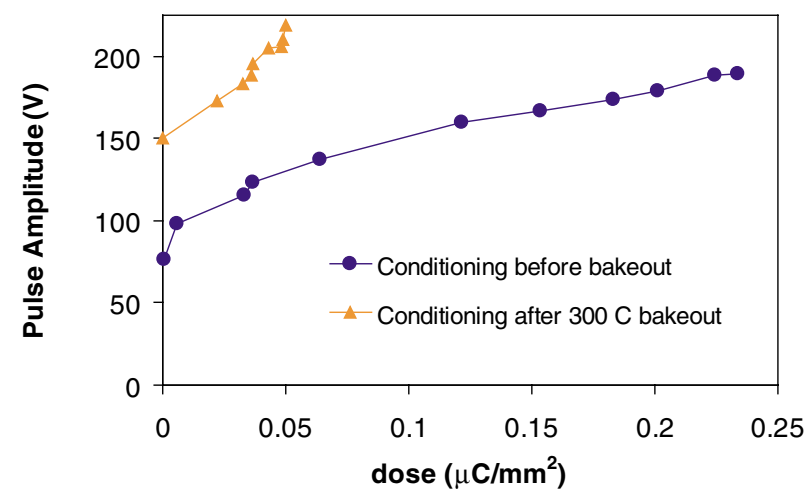

FIG. 3. (Color) Minimum pulse amplitude required to trigger multipacting as a function of electron dose for a stainless steel surface after bakeout (upper curve) and for unbaked stainless steel (lower curve). See Ref. [2] for more details. 
discussed for a long time (see Ref. [5] for more details). Here we are using simultaneous multifrequency operation in the microwave range. This type of operation is also well known for lasers (mode locking), but is not common for microwave circuits.

The conceptual scheme is shown in Fig. 4. The pulse coming from the chamber and the pulse coming from the amplifier are superimposed by means of a directional coupler. The rf pulses coming from the signal generator are amplified in the power amplifier, and then introduced into the wide band directional coupler. Part of the pulse's power is dumped into the rf load, while another part enters the chamber. When leaving the chamber, the pulse again goes through the coupler, where it is added to the next pulse delivered by the amplifier. The $90^{\circ}$ phase shifter between the signal generator and the power amplifier compensates for the $90^{\circ}$ offset introduced by the coupler. The capacitive pickup on top of the chamber is used to evaluate the power enhancement effect of the RR. A measure of $V_{W}$ will be given by means of the resistor probe (RP), included as a part of the ring.

The RR has stringent requirements: low reflection from the traveling wave transmission line and an $\mathrm{rf}$

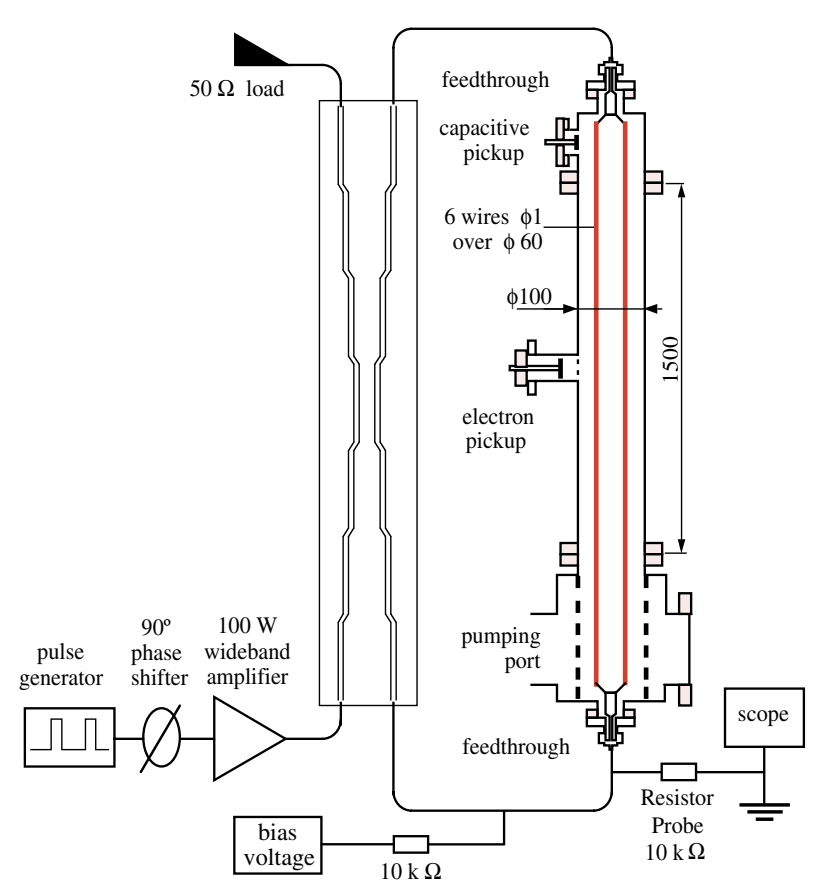

FIG. 4. (Color) Resonant ring outline. Pulses from the signal generator are amplified in the power amplifier and introduced into the wide band directional coupler. Part of the pulse is dumped at the end of one arm of the coupler into the rf load, while the other arm sends the induced signal to the chamber. When leaving the chamber, the pulse enters the coupler again, where it is added to the next pulse delivered by the amplifier. The phase shifter compensates for the $90^{\circ}$ phase offset produced by the coupler. The resistor probe is used to measure the traveling voltage $\left(V_{W}\right)$ through the ring.

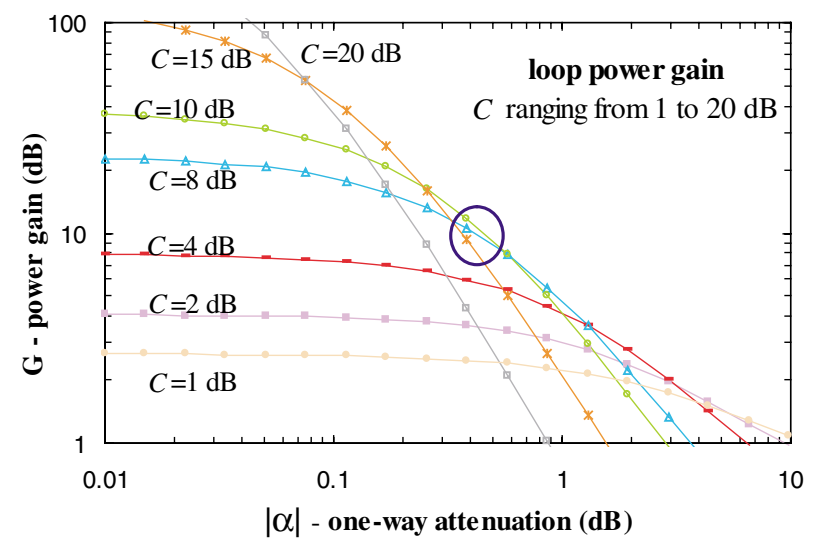

FIG. 5. (Color) Power gain in the resonant ring $(G)$ as a function of the attenuation in the ring $(\alpha)$ and the voltage coupling factor $C$. The circle marks a possible choice of parameters: $|a| \sim 0.5 \mathrm{~dB}$ and $C \sim 10 \mathrm{~dB}$ to get a power gain $G$ around $9 \mathrm{~dB}$.

coupler that has to be designed ad hoc. The final goal is to achieve a gain for the incident power of around 8 or $9 \mathrm{~dB}$, i.e., nearly 10 times the amplifier output power. In a $\mathrm{RR}$, the (maximum) loop power gain $(G)$ is given by [5]

$$
G=\left[\frac{C}{1-10^{-|\alpha| / 20} \sqrt{1-C^{2}}}\right]^{2},
$$

where $C$ is the voltage coupling factor of the coupler and $\alpha$ is the one-way attenuation in the ring (in $\mathrm{dB}$ ). Equation (1) gives $G$ in $\mathrm{dB}$ when $\alpha$ is expressed in $\mathrm{dB}$, and $C$ is expressed in its linear form. Figure 5 sketches the power gain as a function of the attenuation for different values of $C$. To obtain a useful gain $(G \sim 9 \mathrm{~dB})$, a possible choice is $|\alpha| \sim 0.5 \mathrm{~dB}$, and $C \sim 10 \mathrm{~dB}$ (circle in Fig. 5).

Those conditions have to be maintained up to a maximum frequency $f_{\max }$ given by the relative bandwidth (BW) of the coupler and by the minimum working frequency $\left(f_{\min }\right)$ of the setup. As seen in Table $\mathbf{I}$, the bunch spacing in LHC will be $25 \mathrm{~ns}(40 \mathrm{MHz})$. In order to have some "contingency margin" $f_{\min }=30 \mathrm{MHz}$ has been chosen as the lowest cutoff for the coupler. Different LHC operating scenarios with $50 \mathrm{~ns}$ bunch spacing are under study [6]. Such a low $f_{\min }$ allows our setup to provide reliable (not exact) information even for this last case, which would imply frequencies as low as $20 \mathrm{MHz}$. A BW equal to about 20 is the maximum possible for this kind of coupler, it being limited by practical implementation related difficulties. Thus, $f_{\max }=\mathrm{BW} * f_{\min }=600 \mathrm{MHz}$. The one-way attenuation $\alpha$ depends mainly on the reflection coefficient and the transmission losses in the TW chamber (Sec II), while $C$ is a specification of the coupler (Sec. III).

\section{IMPROVEMENTS ON THE TRAVELING WAVE CHAMBER}

Reducing the one-way attenuation $(\alpha)$ requires acting both on the transmission losses of the six wires in the 


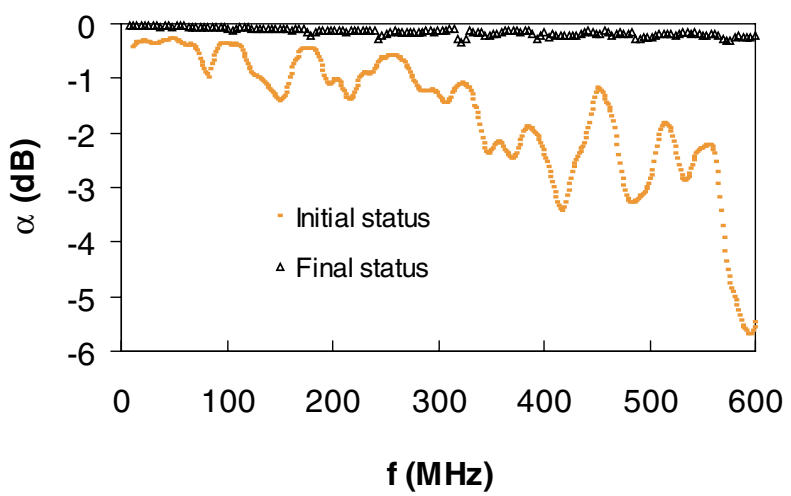

FIG. 6. (Color) Transmission coefficient of the TW chamber before (orange segments) and after (black triangles) the improvements described in Sec. II. Finally, the transmission coefficient is within desired limits: $-0.5 \mathrm{~dB}$ up to $600 \mathrm{MHz}$.

vacuum chamber and on the impedance matching among cables, feedthroughs, and the coaxial structure (i.e., reducing reflections). The frequency response of the initial setup has been measured with a vector network analyzer HP8753D (VNA), as shown in Fig. 6 (transmission coefficient versus frequency) and Fig. 7 (characteristic impedance, $Z_{\text {line }}$, along the structure). These plots compare the initial situation (orange segments) to the improved one (black triangles). The initial transmission coefficient is plotted in Fig. 6 (orange segments), which shows that the coefficient needs to be further reduced (at least up to $f_{\max }$ ). Using the time domain option (step mode) of the instrument, the reflection coefficient $\Gamma$ can be measured as a function of the position along the coaxial line. Then from

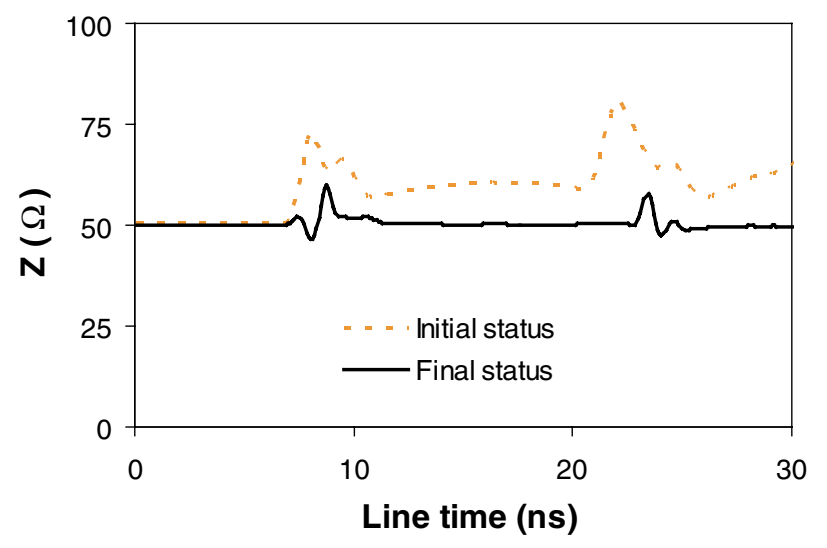

FIG. 7. (Color) Characteristic impedance along the chamber before (orange segments) and after (black triangles) the improvements described in Sec. II. Eventually, the impedance along the line is close to $50 \Omega$ everywhere except for the unavoidable residual mismatches after the feedthroughs (transition from the feedthroughs to the six wire structure), which do not significantly affect the transmission coefficient.

$$
Z_{\text {line }}=Z_{0} \frac{1+\Gamma}{1-\Gamma}
$$

one gets $Z_{\text {line }}$ along the transmission line, as shown in Fig. 7. Ideally, the characteristic impedance should be $50 \Omega$ all along the path in order to avoid reflections. The orange segments (original status) indicate that there are impedance mismatches at the feedthrough locations as well as along the wire (a minor effect). The two transition pieces, joining the wires to the feedthroughs, are a six armed star and a cylindrical joint (see Fig. 8 for more details). They have been electrically and mechanically designed to provide a smooth rf transition: the joint is made of $\mathrm{Cu}$ and the $\mathrm{Cu}-\mathrm{Be}$ transition star has been gold plated in order to reduce its contact resistance and to improve its welding properties. The diameter of the different parts of the joint is chosen to match the $50 \Omega$ line impedance, given that the impedance $Z$ of a coaxial line with circular cross section is

$$
Z=60 \ln \left(D_{\text {ext }} / D_{\text {int }}\right),
$$

where $D_{\text {ext }}\left(D_{\text {int }}\right)$ is the external (internal) conductor diameter. On top of the chamber, the six $\mathrm{Cu}$ wires are crimped and soldered on the transition star's arms, while on the bottom part they are fixed by clamps. The diameter of the circle formed by the six wires ( $60 \mathrm{~mm}$ in our case) has been optimized following experimental results (no analytical approach is available). However, Eq. (3) shows that a comparable single wire configuration would require a wire $43 \mathrm{~mm}$ in diameter. As mentioned in the introduction, a wire of that diameter would be an obstacle

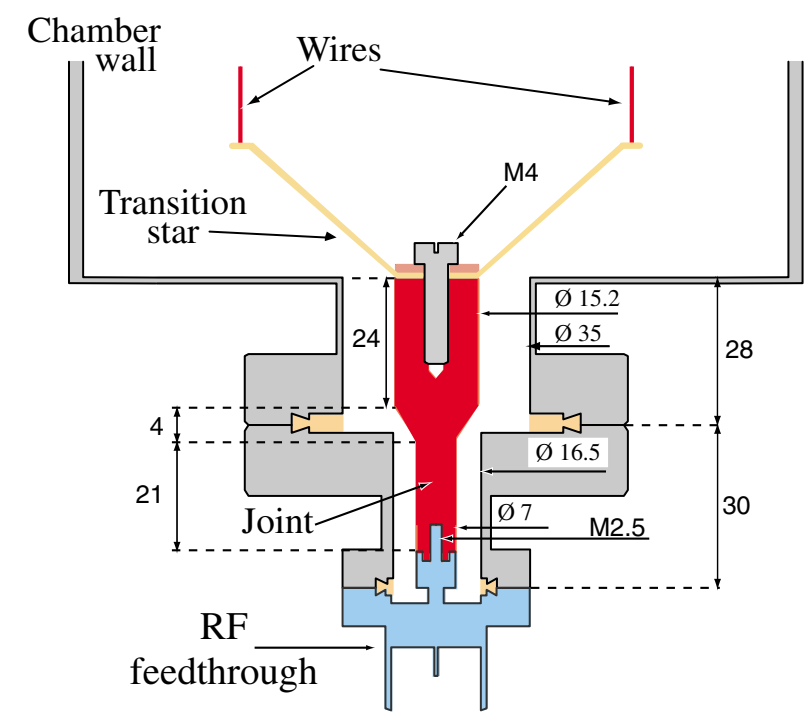

FIG. 8. (Color) Bottom part of the chamber, where we can see the transition pieces between the feedthrough and the six wires. The joint is made of $\mathrm{Cu}$, while the transition star is made on $\mathrm{Cu}-\mathrm{Be}$. The transition star has six arms, corresponding to the six inner wires, but only two of them are drawn here. Lengths are given in $\mathrm{mm}$. 


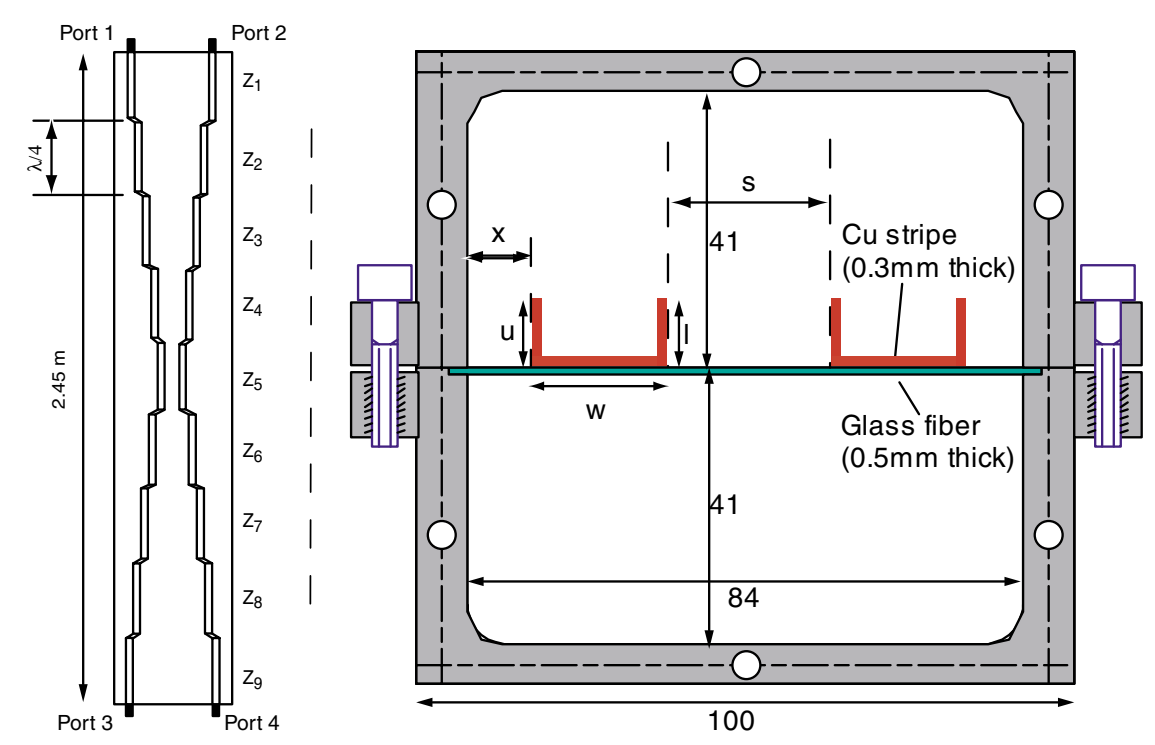

FIG. 9. (Color) Top view (left side) and cross section (right side) of the coupler. The left side shows the layout of the coupler with the nine sections (referred to as $Z_{i}$ with $\left.i=1, \ldots, 9\right)$. Since the coupler is symmetric, $Z_{j}=Z_{10-j}(j=1, \ldots, 5)$. The right picture shows the copper strips' parameters $(w, u$, and $l$ ) and their positions ( $x$ and $s)$. These change for each section in order to obtain the appropriate impedance values. All distances are given in $\mathrm{mm}$.

for electron motion. On the other hand, if a typical wire ( $\sim 3 \mathrm{~mm}$ diameter) were used, $Z$ along the chamber would be about $200 \Omega$, much larger than the desired $50 \Omega$ required for matching. Special gold-plated feedthroughs, ${ }^{2}$ taken from the LEP standing waves feedback cavities (LEP SWC), have been used both for ultrahigh vacuum (UHV) performance and mechanical robustness (in order to resist the mechanical tension of the wires). See Ref. [7] for more details about the design of this joint.

All these improvements produced the effect seen in Figs. 6 and 7 (black triangles). Since the impedance is close to $50 \Omega$ all along the line, except at the unavoidable transition between the six wires structure and feedthroughs, the transmission coefficient is now within the correct limits (less than $0.5 \mathrm{~dB}$ up to $600 \mathrm{MHz}$ ). According to the measurements, this residual mismatch causes no significant loss in $\alpha$. See Ref. [7] and the Appendix for more details about both the rf parameters of the chamber and the mechanical improvements to it.

\section{COUPLER DESIGN}

As stated above, the coupler should have a voltage coupling factor $C=10 \mathrm{~dB}$ in the whole frequency range. Moreover, the coupler must be able to tolerate dc isolation up to $1 \mathrm{kV}$ between the strip lines and ground. The dc isolation of the central wires is needed in order to simulate the bunched beam's electric fields: the central voltage must be zero (between bunches) or positive (presence of a bunch). As the rf pulses coming from the amplifier are

\footnotetext{
${ }^{2}$ Quartex A12 O3, ceramic isolation $Z_{\text {nominal }}=50 \Omega$.
}

positive with a negative base line, a positive bias on the order of half of the pulse's amplitude is needed to set the base voltage to zero. This bias (one to several hundred volts) depends on the pulse's amplitude and duty cycle. One $\mathrm{kV}$ insulation gives a proper safety margin.

As seen in Sec. I, the lowest cutoff $\left(f_{\min }\right)$ has been fixed to $30 \mathrm{MHz}$. However, since the aim is to simulate LHC bunches, where the bunch spacing can go up to $\Delta T=$ $50 \mathrm{~ns}$, the lowest relevant frequency $\left(f_{L}\right)$ is $f_{L}=1 / \Delta T=$ $20 \mathrm{MHz}$ (with reduced performance). The upper frequency limit $\left(f_{\max }\right)$ is set to $600 \mathrm{MHz}$ as explained in Sec. I. The $\lambda / 4$ symmetric nine section coupler described in Ref. [8] accomplishes our requirements. Since the central frequency is $300 \mathrm{MHz}$, each section is $\lambda / 4=$ $25 \mathrm{~cm}$, which implies a coupler length $\sim 2.25 \mathrm{~m}$ (see Fig. 9). Because of the nonstandard specifications, the coupler has been built ad hoc using $0.3 \mathrm{~mm}$ thick copper strips (to reduce Ohmic losses) and bending them as shown in Fig. 9 (right picture). Such a "U-like shape" is repeated for each section, varying its characteristic lengths: $x, w, u, l$, and $s$. The dimensions of the shielding box are chosen according to the cutoff frequency of the high order-propagating mode (i.e., $1 \mathrm{GHz}$ for our structure).

The voltage coupling factor $(C)$ depends on the characteristic impedance of the odd and even TEM propagating modes $\left(Z_{\text {odd }}\right.$ and $\left.Z_{\text {even }}\right)$. The free design parameters are the geometrical dimensions defined in Fig. 9. They are carefully chosen to meet the required value of $Z_{\text {odd }}$ and $Z_{\text {even }}$ for each section, as tabulated in Ref. [9].

The procedure used to build each section is as follows: For a given geometry (i.e., a set of values for $x, w, u, l$, and $s$ ), the section's impedances $\left(Z_{\text {odd }}^{j}\right.$ and $Z_{\text {even, with } j=}^{j}=$ 
$1, \ldots, 9)$ are first computed with SUPERFISH, a 2D electrostatic computer code widely used in rf accelerating cavities [10]. Since the coupler is symmetric, $Z_{j}=Z_{10-j}$ $(j=1, \ldots, 5)$. Then, the section's impedances are measured on a test stand. The test stand is a dedicated $50 \mathrm{~cm}$ long coupler with precisely the same cross sectional dimensions. These are the only dimensions affecting $Z_{\text {odd }}$ and $Z_{\text {even }}$. The two $\mathrm{Cu}$ strips (50 $\mathrm{cm}$ long and with the cross sectional dimensions previously calculated) forming section $j$ are put on the test stand, and $Z_{\text {odd }}^{j}$ and $Z_{\text {even }}^{j}$ are then measured using the VNA with the help of a hybrid coupler. They are measured in the same way that we will measure $Z_{\text {odd }}$ and $Z_{\text {even }}$ for the total coupler (see below). Once the measurements of these strips fit the desired values for $Z_{\text {odd }}^{j}$ and $Z_{\text {even }}^{j}$, the strips are cut into two $25 \mathrm{~cm}$ long sections that will each become part of the final coupler as $Z_{j}$ and $Z_{10-j}$.

For the section design, the SUPERFISH simulation works as follows: Either the two strips are both (numerically) excited with a voltage $+V$ (even mode), or one is excited with $+V$ and the other with $-V$ (odd mode). The code computes the energy stored inside the box for each excitation ( $U_{\text {odd }}$ and $U_{\text {even }}$ ). Following the analogy with electric circuits [11], one gets

$$
Z_{\text {odd }}=\frac{2 V^{2}}{c U_{\text {odd }}}
$$

and

$$
Z_{\text {even }}=\frac{V^{2}}{c 2 U_{\text {even }}}
$$

where $c$ is the velocity of light.

Figure 10 shows the impedance values along the coupler for each mode. The ideal (or theoretical) impedance values found in Ref. [9] are marked with red crosses. In Fig. $10, Z_{\text {odd }}$ and $Z_{\text {even }}$ are measured by connecting the VNA to ports 1 and 2 (Fig. 9, left) through a hybrid coupler to give a phase offset: $0^{\circ}$ for even mode measure-

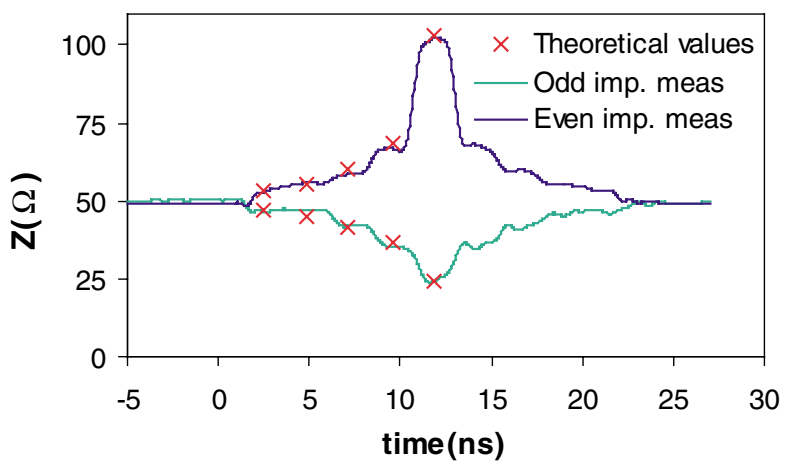

FIG. 10. (Color) $Z_{\text {odd }}$ and $Z_{\text {even }}$ measured in the final coupler as a function of the time (i.e., position along the line). Measurements from the second half of the structure are not valid because of reflections.

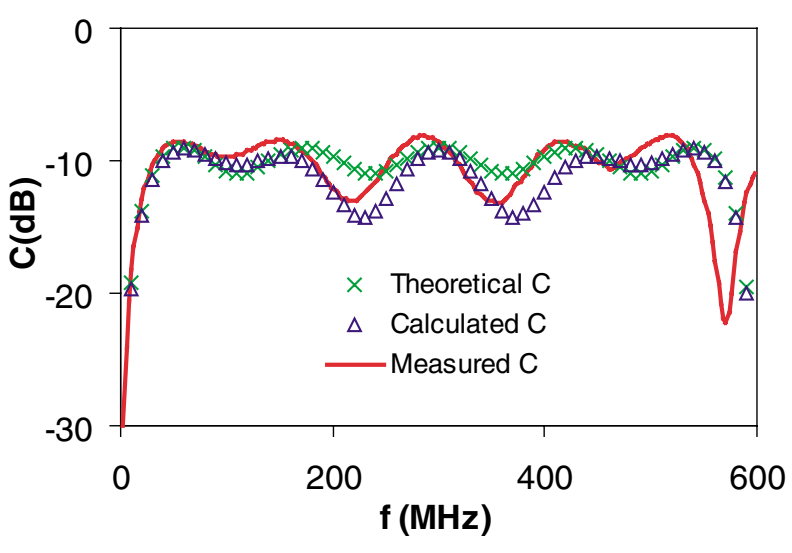

FIG. 11. (Color) Coupling factor of the final coupler. The ripple of the measured coupling factor (red line) is reasonably close to both the theoretical (green crosses) and calculated (blue triangles) behavior in the relevant frequency range (20 to $530 \mathrm{MHz}$, as explained in the text).

ment, $180^{\circ}$ for the odd mode measurement. The time domain (step mode) reflection data are then converted to impedance data using Eq. (2). The data for the second half of the structure are not valid because of multiple reflections. Results from similar measurements using ports 3 and 4 (Fig. 9, left) are the same to within minor tolerances.

Figure 11 shows the behavior of $C$ as a function of frequency for three cases. The first case is the theoretical one, which corresponds exactly to the theoretical impedance values given in Ref. [9] (traced with green crosses). The second is the calculated behavior of $C$ from $Z_{\text {odd }}$ and $Z_{\text {even }}$ measured for each section separately (blue triangles). The third is the measurement along the entire coupler using the VNA (red line). The calculated value of the coupling factor $C$ in Fig. 11 (blue triangles) was computed with numerical simulations of the coupler using commercial codes (ENSEMBLE and SERENADE ${ }^{3}$ ) designed for high speed circuits, but suitable for computing $S$ parameters and full-wave fields for microstrip and planar microwave structures. The red curve is the transmission between ports 1 and 2 of the coupler. The actual working range of the coupler is from 20 to $530 \mathrm{MHz}$ instead of from 30 to $600 \mathrm{MHz}$. The effects of this difference are negligible in the final performance of the RR. The ripple in $C$ is sensitive to small variations in the characteristic impedance of each section. See the Appendix for more information about the rf parameters of this coupler.

\footnotetext{
${ }^{3} \mathrm{~A}$ portion of the geometry is solved with ENSEMBLE to obtain the $S$ parameters; then the full structure is simulated in SERENADE as microwave cascaded networks. Both softwares are developed by Ansoft, and references are available at http://www.ansoft.com.
} 


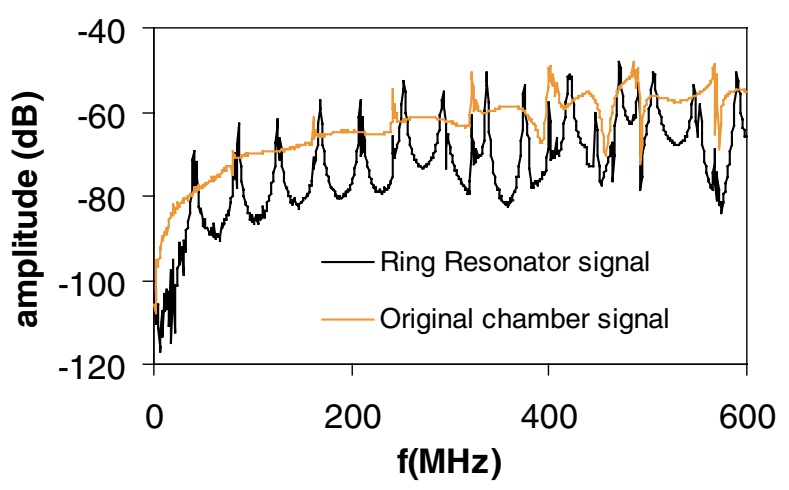

FIG. 12. (Color) Amplitude of the signal seen in a button pickup on the top of the chamber as a function of frequency. The effect of the RR (black line) is compared with the original chamber (orange line). In this plot, the distance between peaks is slightly greater than $40 \mathrm{MHz}$ because the cable length of the actual RR was not exactly $25 \mathrm{~ns}$.

\section{THE FINAL RESONANT RING}

The final layout of the RR is shown in Fig. 4. In order to evaluate the power enhancement effect of the RR, we measured the transmission between a button probe located on top of the chamber (capacitive pickup at Fig. 4) and the feedthrough at the bottom of the chamber. In other words, we measured the quotient of transmitted signal to incoming signal as a function of frequency (i.e., $S_{21}$ measurement). Figure 12 compares the signals seen using the RR without the phase shifter (black line) to the signal in the original TW chamber (orange line). The power enhancement occurs only at particular frequencies which are integer multiples of $f_{R}=1 / T_{R}$, where $T_{R}$ is the round-trip time in the RR. The $T_{R}$ time also depends on the length of the connecting cables. It was chosen to be $25 \mathrm{~ns}$, the same as the nominal LHC bunch spacing. Figure 11 shows a value of $f_{R}$ close to $40 \mathrm{MHz}$. The difference between the orange line (measured directly to the chamber) and the black curve (measured using the RR) shows a minimum gain at $\left(n^{*} f_{R}\right)$ of $6 \mathrm{~dB}$, where $n$ is a natural number.

To compensate for the $90^{\circ}$ phase offset introduced by the coupler, the pulse has been "predistorted" with a phase shifter ${ }^{4}$ placed just after the source (see Fig. 4). The $90^{\circ}$ phase shifter between the signal generator and the power amplifier has two purposes. First, it eliminates the $90^{\circ}$ phase shift of our main coupler. Second, the phase shifter increases the amplitude of the signal actually sent into the RR. In fact, Fig. 13 shows a Gaussian unitary pulse without any phase shift (orange line) together with the $90^{\circ}$ phase shift of an ideal $90^{\circ}$ phase shifter (black line). The amplitude of the phase shifted pulse varies from -0.65 to +0.65 . Assuming that the amplifier output

\footnotetext{
${ }^{4}$ Manufactured by MITEQ. See Appendix for more details about the phase shifter.
}

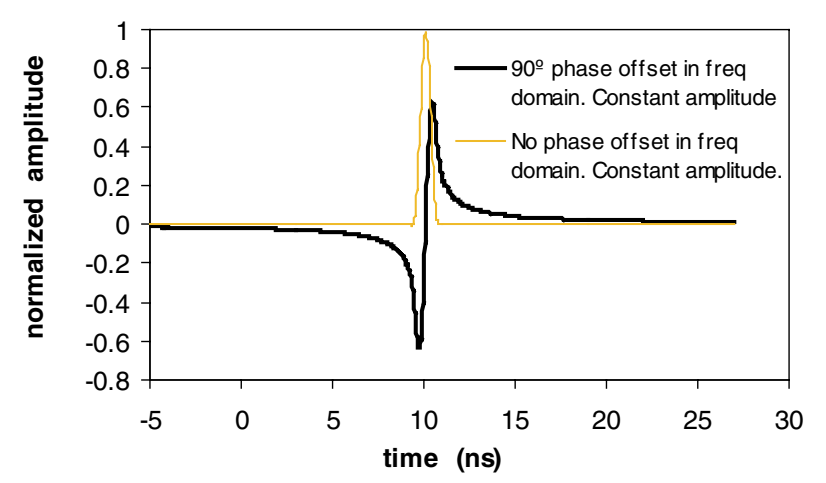

FIG. 13. (Color) The effect of a $90^{\circ}$ phase shift on a Gaussian pulse. The plot shows a Gaussian unitary pulse without any phase shift (orange line) and with a $90^{\circ}$ phase shift (black line), as in an ideal phase shifter. The curves are obtained by measuring the transmission between the input and output port of the shifter with the VNA (time domain, pulse mode).

voltage swing is \pm 1 (after normalization), the "bipolarlike" signal is amplified by $2 / 1.3=1.53$, i.e., $3.7 \mathrm{~dB}$. Thus the total power enhancement is 6 (RR without the phase shifter) plus 3.7 (due to the pulse expansion introduced by the phase shifter), or a total gain of $9.7 \mathrm{~dB}$. This is equivalent to a factor of 3 or more increase in the available TW pulse amplitude.

The circulating peak voltage through the wires $\left(V_{W}\right)$ is measured by means of the RP shown in Fig. 4. The RP is a "homemade" product designed to measure $V_{W}$ without disturbing the signal in the ring. It can withstand a voltage of several $\mathrm{kVs}$. Its attenuation as a function of frequency is shown in Fig. 14. The attenuation is reasonably constant for the relevant frequency range (up to $f_{\max }$ ) and the maximum attenuation is set to $-43.44 \mathrm{~dB}$. Figure 15 shows $V_{W}$ as a function of the peak output signal of the signal generator $\left(V_{S G}\right)$, where $V_{W}$ has been computed using the most pessimistic case (i.e., an attenu-

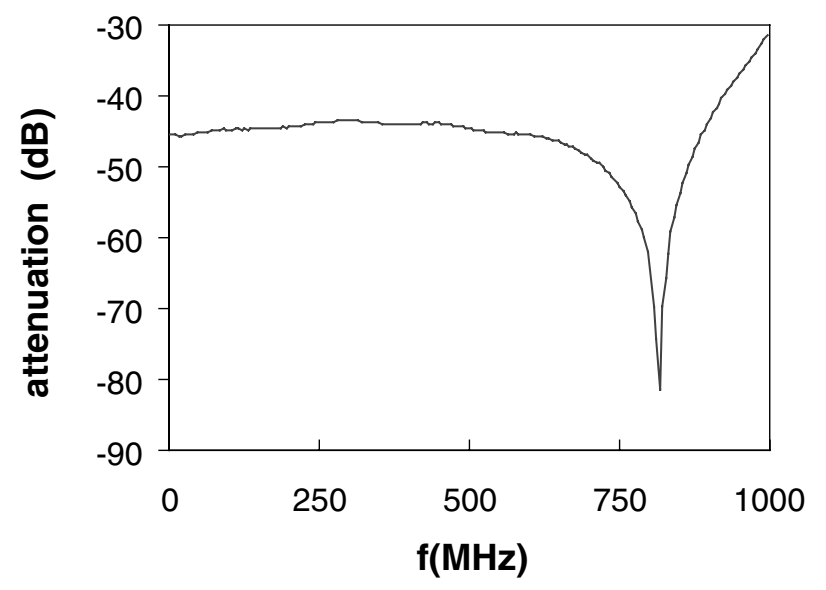

FIG. 14. Attenuation produced by the RP as a function of frequency (measured with the VNA). In the plateau, the average value is $-45 \mathrm{~dB}$ and the maximum is $-43.44 \mathrm{~dB}$. 


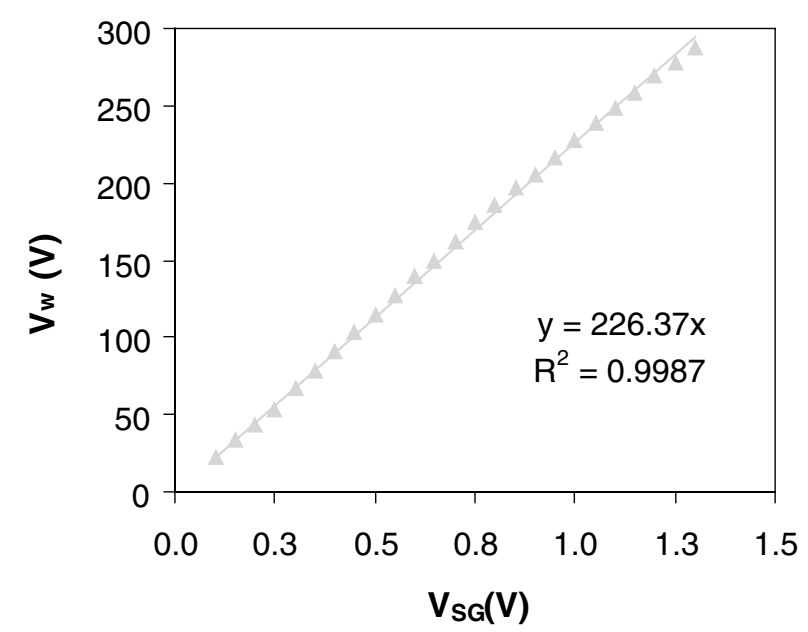

FIG. 15. RR behavior as a function of the peak voltage given by the signal generator $\left(V_{S G}\right)$. The green triangles represent the calculation of the voltage value taking into account an attenuation for the RP of $-43.44 \mathrm{~dB}$. As can be seen from the $R$-squared value, the voltage at the RP varies linearly within the range of $V_{S G}$. The measurement does not go beyond $V_{S G}=$ $1.3 \mathrm{~V}$ because multipacting is triggered.

ation of $-43.44 \mathrm{~dB}$ ). The data stop at $V_{S G}=1.3 \mathrm{~V}$ because multipacting is triggered. The pressure then increases due to electron induced desorption from $10^{-8}$ to above $10^{-6}$ mbar. It takes a few seconds for this pressure rise to occur. Once it has occurred, the experiment is stopped. If the experiment was not stopped the pressure would continue to rise possibly causing gas breakdown or other effects such as glow discharge. The vacuum pump would also trip off (its nominal limit is $10^{-5} \mathrm{mbar}$ ). The amplifier starts clipping (its linear response is lost) for $V_{S G}=1.75 \mathrm{~V}$ which, according to the relation marked in Fig. 15, corresponds to $V_{W}=390 \mathrm{~V}$. However, linearity cannot be fully guaranteed for $V_{S G}>1.3$, and hence the maximum value for $V_{W}$ cannot be determined precisely.

As has been seen in Fig. 12, the pulse amplification takes place when the round-trip time $\left(T_{R}\right)$ is equal to the spacing between two pulses $(\Delta T)$. Despite the fact that it was designed to work for $\Delta T=25 \mathrm{~ns}$, this value can change depending on the cable length (i.e., the ring length). A typical shape for the traveling pulses is shown on Fig. 16. In this case, the available cable length produces $\Delta T=26.6 \mathrm{~ns}$, which is not very far from the $25 \mathrm{~ns}$ bunch length of the LHC. Two waves are present in the ring: the main wave, which has a periodicity of $26.6 \mathrm{~ns}$ and is consistent with the design of the RR, and a smaller reverse circulating wave due to reflections. This small reverse wave was foreseen. It is unavoidable and its final amplitude is not enough to inhibit multipacting. Simulation of LHC bunches are less realistic with this reflected wave, and its consequences have still to be evaluated quantitatively. The pulse width $\left(t_{w}\right)$ was always fixed to $3.1 \mathrm{~ns}$, the minimum available from the amplifier.

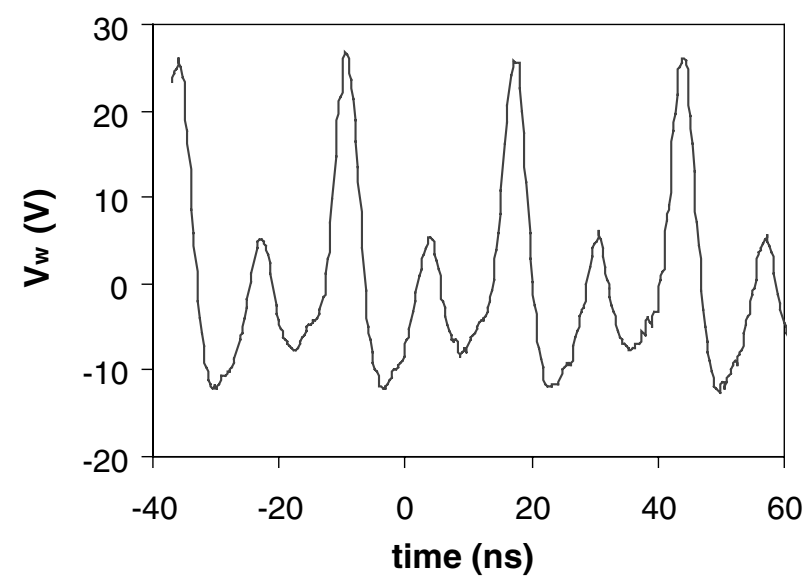

FIG. 16. Voltage amplitude detected in the RP as a function of time. The significance of the two sets of peaks is as follows: The larger peaks are due to pulse addition, and the smaller peaks are the result of the unavoidable reflections, which circulate in the opposite sense $\left(\Delta T=26.6 \mathrm{~ns}, t_{w}=3.1 \mathrm{~ns}\right)$.

This is about 10 times longer than the $0.25 \mathrm{~ns}$ pulse width of the LHC beam. However, rather than reducing the multipacting effect, the longer pulse width intensifies it. If $E_{\min }$ is the minimum electron energy required to trigger multipacting, Ref. [7] sets the minimum peak voltage in the wires $\left(V_{W 0}\right)$ to trigger multipacting as

$$
V_{W 0} \geq \frac{0.51 E_{\min }}{t_{w}} \Delta T \text {. }
$$

The shorter $t_{w}$ is, the higher the $V_{W}$ needed to accelerate electrons enough to trigger multipacting, and vice versa. Since reproducing the electric field produced by an LHC bunch in the laboratory is not possible, sending (Gaussian) pulses of $3.1 \mathrm{~ns}$ in length instead of $0.25 \mathrm{~ns}$ makes the process easier. Equation (6) also shows that sending pulses at $26.6 \mathrm{~ns}$ instead of $25 \mathrm{~ns}$ (from the LHC bunch length) does not influence significantly the multipacting performance. However, in order to properly study the effect of LHC pulses, the cable length should be chosen as close as possible to $25 \mathrm{~ns}$. It is worth mentioning that, from looking at Fig. 16, it is evident that a bias voltage is necessary to ensure that the voltage will always be positive. As shown in Refs. [2,7], the electron energy value has a strong dependence on the parameters of the $\mathrm{RR}$, such as the pulse amplitude $\left(V_{W}\right)$, pulse width $\left(t_{w}\right)$, time between bunches $(\Delta T)$, and bias voltage $\left(V_{b}\right)$. In fact, the $V_{b}$ causes an increase in the electron velocity [2] (i.e., the electron energy).

\section{CONCLUSIONS}

The resonant ring concept has been used in the microwave range in a simultaneous multifrequency mode of operation to upgrade the standard TW setup for electron cloud studies. Improvements on the original TW chamber 
and for the coupler are in good agreement with the initial calculations and objectives. We achieved $|\alpha|<0.5 \mathrm{~dB}$ along the chamber and for the coupler. The ripple of the measured $C$ around $10 \mathrm{~dB}$ is also reasonably close to both the theoretical and calculated behavior in the relevant frequency range. The first results using the RR were successful. Multipacting signatures have been detected in a chamber where the effect was not found before using the original TW setup. The traveling pulses inside the chamber have an amplitude that is significantly larger than it was (up to a maximum of about $390 \mathrm{~V}$ ), which allows for the production of multipacting electrons in the $200 \mathrm{eV}$ range. Preliminary results of the energy spectrum already show evidence for multipacting electrons at $150 \mathrm{eV}$. One of the problems found in the RR is the presence of another parasitic wave caused by unavoidable reflections in the ring. First results show that it does not prevent multipacting in the chamber. The setup is ready for electron cloud studies. The priorities will be to measure the electron energy spectrum as a function of the $V_{W}$, to evaluate the electron cloud buildup time, and to determine whether microwaves can be used as a suitable remedy for multipacting [12]. This setup will be an important tool for studying electron cloud effects and finding remedies since it will allow characterization of the scrubbing effect with different surface treatments. These data can then be compared with results from computer code simulations.

\section{ACKNOWLEDGMENTS}

This work profited from the help of many members of CERN: P. Griessen supplied the feedthroughs from the SWC-LEP; H. Kos and D. Collet precisely machined the pieces we needed; A. Lombardi gave us the idea and helped us with using SUPERFISH; and F. Ruggiero, G. Rumolo, and F. Zimmermann encouraged the authors with their helpful discussions. C. Deibele (ORNL) helped substantially with the calculations of the coupling factor, and M. Pivi (SLAC), K. Zeno, and K. S. Smith (BNL) provided helpful discussions.

\section{APPENDIX: RADIO FREQUENCY ASPECTS OF THE RESONANT RING}

This section is intended for readers interested in the rf analysis of the main parts of the RR. Since the RR is a linear system, it can be fully described by the scattering matrix (S matrix) in the frequency domain. In the following we discuss the matrix components of each part of the RR.

\section{THE TRAVELING WAVE CHAMBER}

The TW chamber itself can be understood as a system with two ports (1 and 2). As in any passive network,

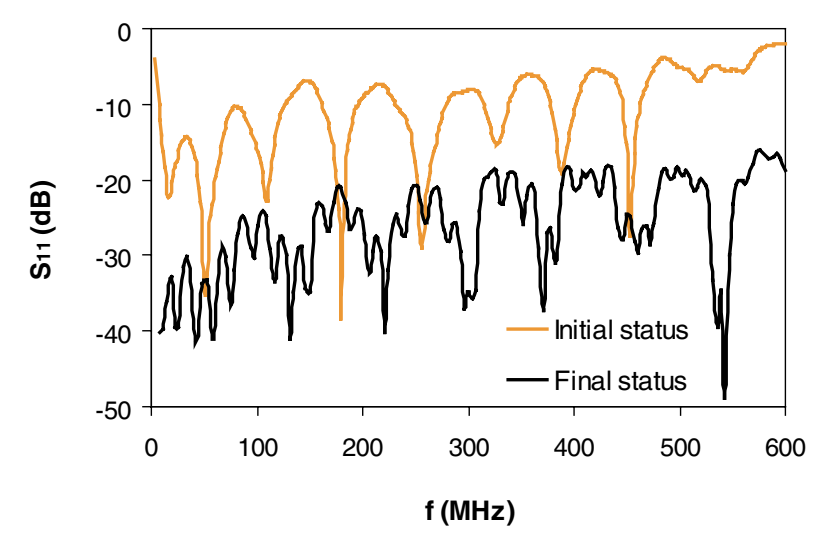

FIG. 17. (Color) Comparison between the initial and final $S_{11}$ coefficient of the TW chamber in the significant frequency range.

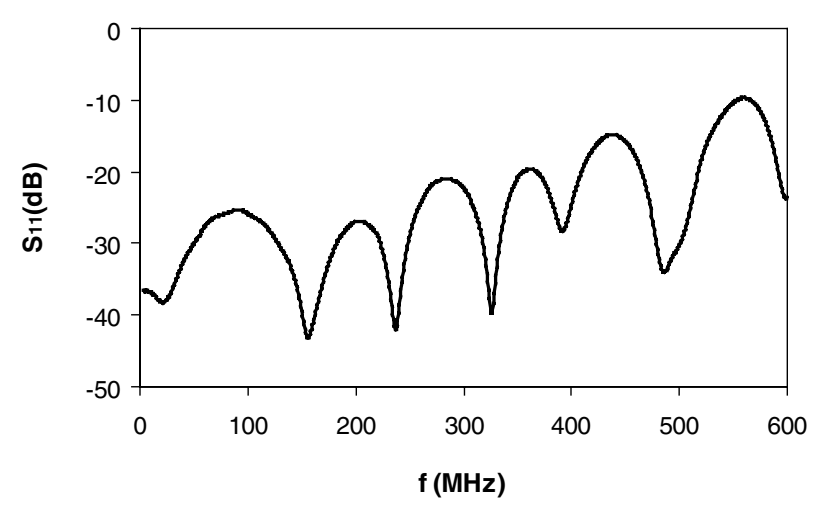

FIG. 18. $S_{11}$ measurement for the manufactured coupler in the relevant frequency range.

symmetric conditions for the TW imply $S_{11}=S_{22}$ and $S_{12}=S_{21}$. Thus, the significant components of the $\mathrm{S}$ matrix are just $S_{11}$ (reflection) and $S_{12}$ (transmission ${ }^{5}$ ). Since $S_{12}$ has already been shown in the text, the only component missing for the complete analysis of this system is $S_{11}$. Furthermore, $S_{11}$ better illustrates the mismatches in the line. The following plot (Fig. 17) shows the comparison between the initial and the final $S_{11}$ coefficient of the TW chamber. It can be seen that the final $S_{11}$ is below $-18 \mathrm{~dB}$ up to $500 \mathrm{MHz}$. This is mainly because the capacitive effects on the top and bottom of the chamber have been reduced with the new transmission piece between feedthroughs and wires. The data has been taken using the VNA.

\section{MAIN PARAMETERS OF THE 10 DB COUPLER}

The coupler is a 4-port system, which means that it requires an $\mathrm{S}$ matrix with 16 components. Symmetric conditions $\left(S_{i i}=S_{j j}\right.$ and $\left.S_{i j}=S_{j i}\right)$ make the system de-

\footnotetext{
${ }^{5}$ Referred to also in the text as $\alpha$.
} 


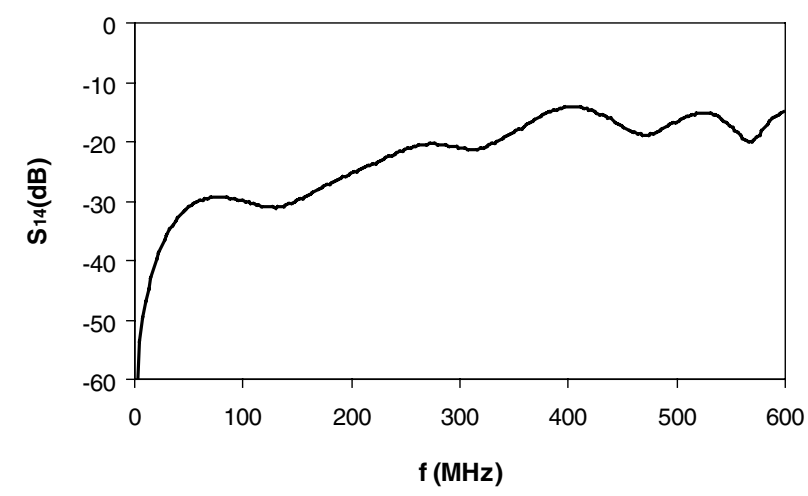

FIG. 19. $S_{14}$ measurement for the manufactured coupler in the relevant frequency range.

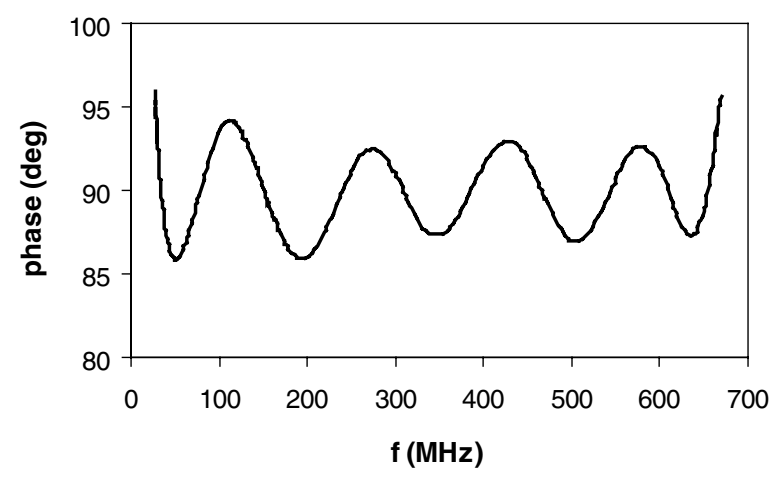

FIG. 20. Phase analysis of the $90^{\circ}$ phase shifter used for the RR.

scribable with just four of them. In order to fully analyze the performance of the coupler, several $S_{i j}$ measurements have been carried out using the VNA. The port numbers are stated in Fig. 9. When an $S_{i j}$ measurement is done, reflectionless terminations are connected to the other ports of the coupler. In the following, we show the $S$ parameters which characterize a directional coupler, according to Ref. [13] . Since $S_{12}$ has been already shown in the text (see Fig. 11), we only show here $S_{11}$ (Fig. 18) and $S_{14}$ (Fig. 19). From $S_{14}$, one can easily calculate the directivity (ratio between the desired- $S_{12}$ - and undesired $-S_{14}$ - signals).

\section{RF ANALYSIS OF THE $90^{\circ}$ PHASE SHIFTER}

The coupler introduces a $90^{\circ}$ phase shift in the signal, which has to be corrected to obtain a reasonable nice signal in the ring. We solved the problem by introducing a $90^{\circ}$ phase shifter (between the signal generator and the amplifier) that changes phase uniformly within the relevant frequency range (30 to $600 \mathrm{MHz}$ ). This piece was provided by MITEQ, and its phase as a function of frequency is shown in the Fig. 20 (data provided by the manufacturer). Experimental results show that the phase ripple in the phase shifter as a function of the frequency (Fig. 20) is not significant and does not affect the performance of the RR.

[1] O. Grobner, in Proceedings of the Particle Accelerator Conference, Vancouver, Canada, 1997 (IEEE, Piscataway, NJ, 1997), pp. 3589-3591.

[2] M. Pivi, Ph.D. thesis, University of Torino, 2000.

[3] V. Baglin, J. Bojko, O. Grobner, B. Henrist, N. Hiilleret, C. Scheuerlein, and M. Taborelli, in Proceedings of the European Particle Accelerator Conference, Vienna, 2000, edited by J. L. Laclar, W. Mitaroff, Ch. PetitJean-Genaz, J. Poole, and M. Regler (Austrian Academy of Science, Vienna, 2000), pp. 217-221.

[4] H. H. Braun, R. Corsini, T. E. D'Amico, J. P. Delahaye, G. Guignard, C. D. Johnson, A. Millich, P. Pearce, A. J. Riche, L. Rinolfi, R. D. Ruth, D. Schulte, L. Thorndahl, M. Valentini, I. Wilson, and W. Wünsch, CERN Yellow Report No. 99-06, edited by R. Corsini, 1999.

[5] G. L. Matthaei, L. Young, and E. M.T. Jones, in Microwave Filters, Impedance-Matching Networks and Coupling Structures (Artech House, Dedham, MA, 1980), Sec. 14.05.

[6] F. Ruggiero et al., in Proceedings of the European Particle Accelerator Conference, Stockholm, 1998, edited by S. Myers, L. Liljeby, and C. Petit-Jean-Genaz (Institute of Physics, London, 1999), pp. 359-361.

[7] U. Iriso Ariz et al., CERN Report No. VAC-TN01-17, 2001, http://lhc-div-vac.web.cern.ch/lhc-div-vac/ VACPAGES/VacuumTechNote/2001/TN_01_17.pdf.

[8] J. R. Harlan Howe, in Stripline Circuit Design (Microwave Associates, Burlington, MA, 1974), Chap. 5.

[9] E. G. Cristal and L. Young, IEEE Trans. Microwave Theory Tech. 13, 557 (1965).

[10] J.H. Billen and L. M. Young, in Proceedings of the Particle Accelerator Conference, Washington, DC, 1993 (IEEE, Piscataway, NJ, 1993).

[11] O. Zinke and H. Brunswig, in Hochfrequenztechnik I (Springer-Verlag, Berlin, 1973), 2nd ed., Vol. I, p. 100.

[12] F. Caspers, F-J. Decker, and F. Zimmerman, in Proceedings of the ECLOUD'02 Workshop, Geneva, 2002, edited by F. Ruggiero, G. Rumolo, and F. Zimmermann (CERN Yellow Report, 2002); see also http://wwwslap.cern.ch/collective/ecloud02/index.html.

[13] F. Caspers, in RF Engineering for Particle Accelerators, edited by S. Turner (CERN, Geneva, 1992), p. 142. 